\title{
Nuclear structure and decay data evaluation in Europe
}

\author{
Alexandru Negret ${ }^{1, a}$, Dimiter Balabanski ${ }^{2}$, Paraskevi Dimitriou ${ }^{3}$, Zoltan Elekes ${ }^{4}$, Theo J. Mertzimekis ${ }^{5}$, Sorin Pascu ${ }^{1}$, \\ and Janos Timar ${ }^{4}$ \\ ${ }^{1}$ Horia Hulubei National Institute for Physics and Nuclear Engineering, Nuclear Physics Department, Măgurele, Romania \\ 2 Horia Hulubei National Institute for Physics and Nuclear Engineering, ELI-NP, Măgurele, Romania \\ 3 Nuclear Data Section, International Atomic Energy Agency, Austria \\ 4 MTA Atomki, Hungary \\ 5 Faculty of Physics, University of Athens, Greece
}

\begin{abstract}
Nuclear Structure and Decay Data (NSDD) activities in Europe include mass-chain and individual nuclei evaluations as well as horizontal evaluations and compilations, data dissemination and educational activities. As such they are essential for a large range of applications from energy, environmental, and medical to basic research in nuclear structure and reactions, all of which are intensively pursued in Europe. Although the NSDD evaluation groups in Europe form part of the international network of NSDD evaluators, which is coordinated by the International Atomic Energy Agency, they are faced with some very distinct challenges. We shortly present the NSDD Data Centre at IFIN-HH, Bucharest and discuss possible actions to improve the situation for the entire European NSDD evaluation effort.
\end{abstract}

\section{Introduction}

The Evaluated Nuclear Structure Data File, ENSDF represents the most important database in the field of nuclear structure. It gathers together the best available knowledge on all isotopes investigated during the last century in nuclear physics experiments. The file is hosted on the website of Brookhaven National Laboratory, USA [1] and on several mirror servers.

ENSDF is maintained and continuously updated and extended by the Nuclear Structure and Decay Data (NSDD) network of evaluators. NSDD is coordinated by the International Atomic Energy Agency (IAEA) and most of the evaluators perform their activity within 16 Data Centres spread worldwide.

The importance of ENSDF cannot be overstated: it constitutes the main source of nuclear structure information required in various fields:

- Practically the entire nuclear structure community uses ENSDF as a professional and reliable source of experimental information.

- Several databases used in other fields of nuclear physics are generated starting from ENSDF. See, for example, the RIPL (Reference Input Parameter Library) database [2] used in nuclear reaction studies.

- Many applications require specific nuclear structure data and ENSDF together with the related horizontal evaluations currently represents the most reliable source of such information. This includes very sensitive data like the nuclear lifetimes which are particularly important for medicine and other applications
We will shortly describe the evaluation-related activities in the second section. Despite the fact that the groups and Data Centres based in Europe are part of NSDD, they face specific challenges. Therefore the third section is dedicated to an overview of the European contribution to this effort with a particular emphasis on the Romanian Data Centre from IFIN-HH (Horia Hulubei National Institute of Physics and Nuclear Engineering). The last section discusses possible actions to improve our participation to NSDD.

\section{Nuclear structure evaluation activities}

\subsection{Mass-chain and individual nuclei evaluations}

The main activity performed by the NSDD members is the compilation and evaluation of the existing experimental information in order to update and extend ENSDF. While evaluations of individual nuclei are often performed, it is usual that all isotopes within a mass chain are evaluated at once when significant new experimental information becomes available. The new mass-chain evaluations are currently published as extended (200-300 pages) peerreviewed articles in Nuclear Data Sheets or Nuclear Physics A.

The work of the evaluator starts with an extensive documentation on all experimental information regarding a nucleus. The Nuclear Science References (NSR) database [3] is used together with other sources. Articles, laboratory reports, $\mathrm{PhD}$ theses, private communications are consulted and grouped on datasets. Each dataset compiles the existing information coming from a certain type of experiments: nuclear decays or various nuclear reactions.

After building all the individual datasets, the actual evaluation of the existing experimental data is performed.

\footnotetext{
a e-mail: alnegret@tandem.nipne.ro
} 


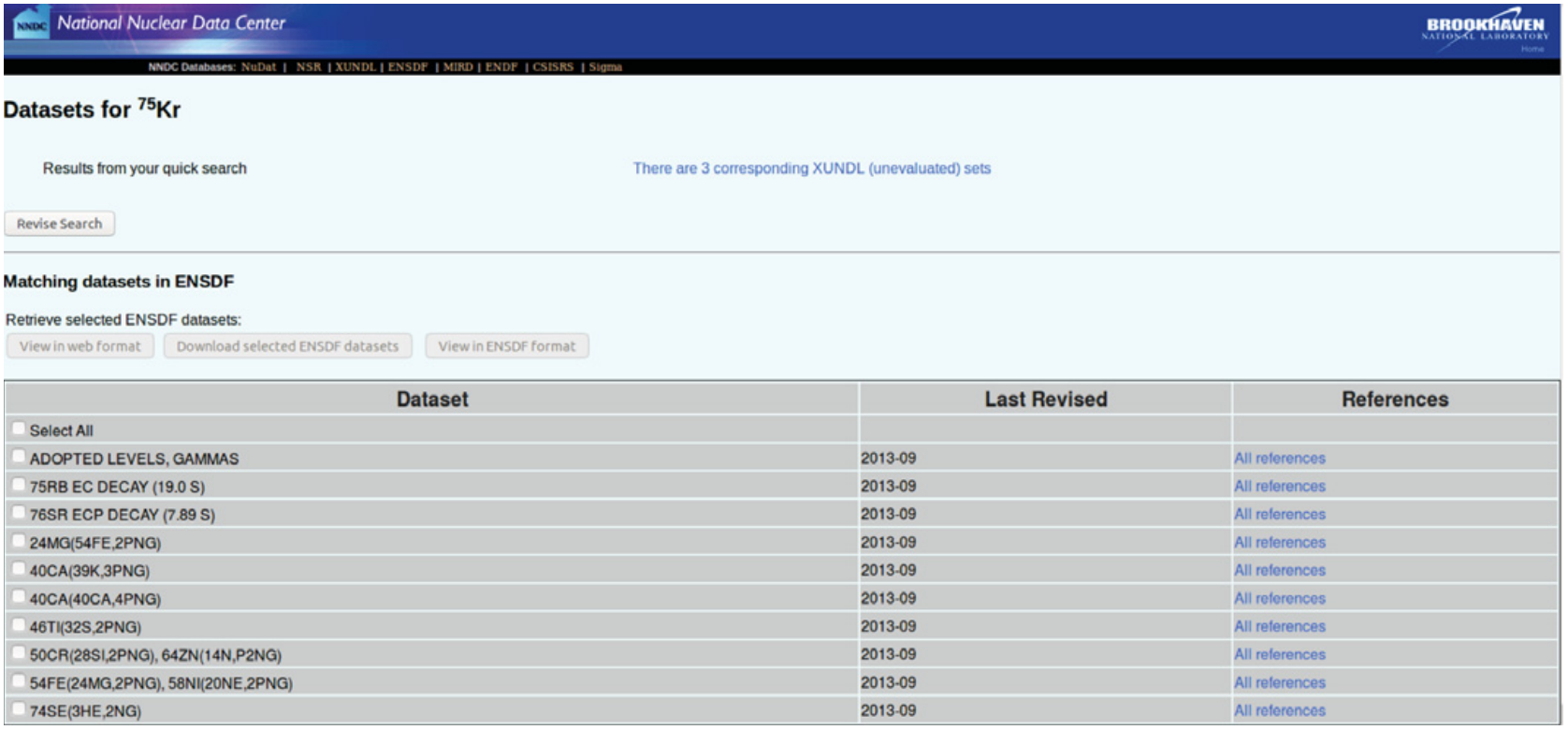

Figure 1. Example of a retrieval from the ENSDF website. The dataset structure of the ${ }^{75} \mathrm{Kr}$ evaluation is visible.

The process involves critical judgment and decision but also the use of various software packages implementing averaging and minimization techniques, consistency checks, etc. Very often discrepant data have to be reconciled or eliminated. The result of the evaluation process is the Adopted levels and gammas dataset that represents the best available information on the level structure, decay modes and transitions of the studied nucleus. Figure 1 shows as an example the structure of the information available for the ${ }^{75} \mathrm{Kr}$ isotope as it is displayed on the ENSDF server from the BNL website. Next to the Adopted levels and gammas dataset, one can notice 2 decay datasets and 7 reaction datasets.

The full evaluation of a mass chain may take up to two years depending on the complexity of the mass chain and the experience and the time involvement of the evaluator.

\subsection{Other activities}

Besides the actual nuclear structure evaluation with the purpose of updating ENSDF, several related activities are also important. This includes horizontal evaluations, data compilation and dissemination, and training activities.

Instead of looking for the experimental information on one nucleus, a horizontal evaluation studies a certain physical quantity for many isotopes from the nuclear chart. Perhaps the most known and widely used such evaluation is the Atomic Mass Evaluation (AME) [4] currently performed by a group of the Institute of Modern Physics from Lanzhou, China.

Data compilation and dissemination is important especially for the impact it has on the scientific community. As an example, we mention here a recent European contribution: the nuclear moments compilation performed by a group from the University of Athens [5] that incorporates data from previous evaluations [6], ENSDF, and recent experiments.

Finally, the training activities have a multi-folded purpose: to spread the information regarding ENSDF and, more generally, the knowledge in the field of nuclear physics, but also to formate new evaluators for ENSDF.
Table 1. Evolution of the number of NSDD Data Centres in time.

\begin{tabular}{lccccc}
\hline & 1981 & 1986 & 1996 & 2008 & 2016 \\
\hline North America & 6 & 6 & 6 & 6 & 7 \\
Europe & $\mathbf{6}$ & $\mathbf{5}$ & $\mathbf{4}$ & $\mathbf{1}$ & $\mathbf{2}$ \\
Russia & 2 & 2 & 2 & 1 & 1 \\
Japan & 1 & 1 & 1 & 1 & 1 \\
China & - & - & 1 & 2 & 2 \\
Rest of the world & 1 & 1 & 1 & 3 & 2 \\
\hline
\end{tabular}

As it will be explained in the next section, this represents a critical issue. We note the long series of Workshops on Nuclear Structure and Decay Data: Experiment, Theory and Evaluation [7] organized every two years by IAEA in collaboration with The Abdus Salam International Centre for Theoretical Physics (ICTP) in Trieste, Italy.

\section{Evaluation in Europe}

As both fundamental and applied nuclear physics research are well developed in Europe, the use of ENSDF database by the European scientists is extensive. This becomes particularly clear when statistics of the number of retrievals from the website are generated.

However, the contribution to the evaluation process does not rise at the same level. Table 1 displays the evolution in time of the number of NSDD Data Centres worldwide [8]. It shows that, although the number of Data Centres in US and Europe in the eighties were equal, the European contribution decreased drastically afterwards. This happen through the retirement of practically all experienced evaluators. Two new Data Centres were established recently: one in Debrecen (2013) and one in Bucharest (2015). Even if a limited additional evaluation effort is made by small groups in Turkey, Bulgaria and Greece, we consider that this is clearly not sufficient to compensate for the retirements that occurred during the last three decades.

Finally, Table 1 does not reveal a second major concern of the NSDD community: that a significant number of experienced evaluators from North America are currently 
at the post-retirement age. Therefore the major risk exist that, during the next decade, the manpower contribution to ENSDF will drop even further. As a consequence, the information from this major database may become progressively obsolete while the quality of evaluations may significantly decrease.

\section{The data centre at IFIN-HH}

Following a few years of activity in the field and after the competion of two full mass chain evaluations in collaboration with an experienced evaluator $[9,10]$, the group from IFIN-HH became in 2015 one of the NSDD Data Centres. Two scientist dedicate $20 \%$ of their working time to this activity.

The Bucharest Data Centre is responsible for the evaluation of the nuclei with $\mathrm{A}=57-59$ and 117-118. However, contributions to the evaluation of other mass chains are undergone. We mention here the ongoing colaboration between the Data Centres in Bucharest and Debrecen for the evaluation of the $\mathrm{A}=101$ mass chain.

The Data Centre is mainly supported by IFIN-HH while Research Contracts were issued by IAEA to complement this support for a limited period of time.

\section{Perspectives and conclusions}

We argued in the previous section that the European contribution to NSDD has to increase in order to compensate for the retirement of several experienced evaluators during the last decades. Unless the evaluationrelated activities in Europe will increase, the danger exist that the database will gradually loose its importance for the scientific community by becoming obsolete.

However we believe that, complementary to the mass chain evaluation, other related activities can and should be carried by the European scientific community. This includes data compilation and dissemination, training and education activities that widen the use of the database while preparing a new generation of evaluators and, more generally, of nuclear scientists.

In order to coagulate the European effort, we intend to propose to the community a Memorandum of Understanding (MoU) for the creation of the European Nuclear Structure and Decay Data Network (ENSDD). This would give various institutes the opportunity to show their support for the nuclear structure evaluation and compilation effort and to get actively involved in the process.

Also, we propose the inclusion of the nuclear structure evaluation among the activities supported by the Nuclear Physics European Collaboration Committee (NuPECC) of the European Science Foundation [11] in their Long Range Plan.

Finally, we wish to make the European scientific community aware that, on the medium and long term, the research in the field of nuclear structure should integrate the compilation and evaluation efforts. In other words, as the quantity of data increases continuously, the experimental effort has to be complemented by evaluation, so that the results are synthesized, compiled, evaluated, recorded and made available to the community in a standard, easy-to-use format.

The authors thank Dr. Balraj Singh for useful discussions. The scientific support offered by the IAEA during the beginning of the evaluation activity in IFIN-HH and MTA Atomki is acknowledged. TJM acknowledges support by the UoA Natural Sciences Dean's Office.

\section{References}

[1] www.nndc.bnl.gov/ensdf

[2] R. Capote et al., Nucl. Data Sheets 110, 3107-3214 (2009)

[3] B. Pritychenko, E. Bĕták, M.A. Kellett, B. Singh, and J. Totans, Nucl. Instrum. Meth in Phys. Res. A 640, 213-218 (2011)

[4] G. Audi, M. Wang, A.H. Wapstra, F.G. Kondev, M. MacCormick, X. Xu, and B. Pfeiffer, Chinese Physics C 36, 1287-1602 (2012)

[5] T.J. Mertzimekis, K. Stamou, and A. Psaltis, Nucl. Instrum. Meth in Phys. Res. A 807, 56-60 (2016)

[6] N.J. Stone, IAEA Report INDC(NDS)-0658 (2014)

[7] http://indico.ictp.it/event/7641/

[8] D. Balabanski and A.L. Nichols, IAEA Report INDC(NDS)-0543 (2008)

[9] A. Negret and B. Singh, Nucl. Data Sheets 114, 841 (2013)

[10] A. Negret and B. Singh, Nucl. Data Sheets 124, 1 (2015)

[11] www . nupecc.org 